\title{
Experimental evolution of parasitoid infectivity on symbiont-protected hosts leads to the emergence of genotype-specificity
}

Romain Rouchet $^{1,2}$ and Christoph Vorburger ${ }^{1,2}$

1 Institute of Integrative Biology, ETH Zürich, Zürich, Switzerland

2 EAWAG, Swiss Federal Institute of Aquatic Science and Technology, Dübendorf,

Switzerland

Correspondence: Romain Rouchet, Institute of Vertebrate Biology, Academy of Sciences

of the Czech Republic, Kvetna 8, 60365 Brno, Czech Republic, Tel: +420 543422522

E-mail: romain.rouchet@gmail.com

This document is the accepted manuscript version of the following article:

Rouchet, R., \& Vorburger, C. (2014). Experimental evolution of parasitoid infectivity on symbiont-protected hosts leads to the emergence of genotype specificity. Evolution, International Journal of organic Evolution, 68(6), 1607-1616. http://doi.org/10.1111/evo.12377 


\begin{abstract}
:
Host-parasitoid interactions may lead to strong reciprocal selection for traits involved in host defense and parasitoid counter-defense. In aphids, individuals harboring the facultative bacterial endosymbiont Hamiltonella defensa exhibit enhanced resistance to parasitoid wasps. We used an experimental evolution approach to investigate the ability of the parasitoid wasp Lysiphlebus fabarum to adapt to the presence of $H$. defensa in its aphid host Aphis fabae. Sexual populations of the parasitoid were exposed for eleven generations to a single clone of $A$. fabae, either free of $H$. defensa or harboring artificial infections with three different isolates of $H$. defensa. Parasitoids adapted rapidly to the presence of $H$. defensa in their hosts, but this adaptation was in part specific to the symbiont isolate they were evolving against and did not result in an improved infectivity on all symbiont-protected hosts. Comparisons of life history traits among the evolved lines of parasitoids did not reveal any evidence for costs of adaptation to $H$. defensa in terms of correlated responses that could constrain such adaptation. These results show that parasitoids readily evolve counter-adaptations to heritable defensive symbionts of their hosts, but that different symbiont strains impose different evolutionary challenges. The symbionts thus mediate the host-parasite interaction by inducing line-by-line genetic specificity.
\end{abstract}

\title{
KEYWORDS:
}

Aphis fabae, adaptation, facultative symbionts, Hamiltonella defensa, host-parasitoid coevolution, trade-off. 


\section{RUNNING TITTLE:}

Experimental evolution of parasitoid infectivity.

\section{DATA ARCHIVE LOCATION:}

Data will be updated in DRYAD.

\section{Introduction}

Parasitism is the most common lifestyle on Earth and affects nearly all living organisms (Windsor 1998). Host-parasite interactions may lead to intense and continuous processes of reciprocal adaptation for the traits involved in the outcome of the interaction, such as host resistance and parasite virulence (Thompson 1994). These antagonistic coevolutionary dynamics have been widely studied according to their importance in diverse fields such as evolutionary biology, agricultural science, conservation biology or medical science (Woolhouse et al. 2002; Ebert 2008; Laine 2009; Brown and Tellier, 2011). Because the outcome of their interaction is always fatal for one of the antagonists, host-parasitoid systems exhibit particularly strong reciprocal selection and thus offer great scope for the study of coevolution (Godfray 1993; Kraaijeveld et al. 1998).

Aphids frequently rely on facultative bacterial endosymbionts for defense against natural enemies (Oliver et al. 2010). In addition to their primary symbiont Buchnera 
aphidicola, which provides them with essential nutrients missing from their phloem diet, aphids may harbor a large variety of facultative or secondary endosymbionts. These are generally not essential for survival and reproduction (Oliver et al. 2010), but they can provide aphids with important ecological benefits. A particularly common way for these facultative symbionts to increase their frequency within host populations is to provide them with protection against their natural enemies (Oliver et al. 2013). For example, at least four species of symbionts can increase the resistance to fungal pathogens in the pea aphid (Scarborough et al. 2005; Łukasik et al. 2013), and Hamiltonella defensa has been shown to provide different aphid species with protection against parasitoid wasps (Oliver et al. 2003; Ferrari et al. 2004; Oliver et al. 2005; Vorburger et al. 2009). Hamiltonella defensa occurs in approx. 14\% of aphid species (Oliver et al. 2010), it is abundant in natural populations of the pea aphid, Acyrthosiphon pisum (Ferrari et al. 2012; Russell et al. 2013), as well as the black bean aphid, Aphis fabae (Vorburger et al. 2009). The protection against parasitoids afforded by $H$. defensa is influenced by the presence of lysogenic bacteriophages called APSE that are found within the $H$. defensa genome (Moran et al. 2005; Degnan and Moran 2008; Oliver et al. 2008, Oliver et al. 2009). Different strains of $H$. defensa are associated with different variants of this bacteriophage encoding for a variety of toxins targeting eukaryotic cells (Moran et al. 2005), and there is evidence that different strains of $H$. defensa provide different levels of protection against parasitoids (Oliver et al. 2005). Moreover, some studies suggest that resistance conferred by $H$. defensa acts more specifically than the (limited) defenses of $H$. defensafree hosts (Vorburger et al. 2009; Rouchet and Vorburger 2012; Schmid et al. 2012; Cayetano and Vorburger 2013). Hence, the presence of $H$. defensa in aphids may impose 
strong and potentially multifarious selection on parasitoids to adapt to symbiontconferred resistance. Different asexual lines of the parasitoid Lysiphlebus fabarum were shown to exhibit large differences in their ability to overcome H.defensa-mediated defenses of their host, A. fabae, suggesting that parasitoid populations possess the necessary genetic variation for adaptation (Rouchet and Vorburger 2012). Indeed, Dion et al. (2011) showed, using an experimental evolution procedure, that the parasitoid wasp Aphidius ervi can adapt very quickly to the presence of $H$. defensa in the pea aphid $A c$. pisum. Still unknown is whether parasitoid adaptation to one $H$. defensa strain generally improves the ability to overcome symbiont-conferred defenses, whether such adaptation reduces infectivity on $H$. defensa-free aphids, or whether it is constrained by other tradeoffs, e.g. with life-history traits. In this study, we addressed these questions with an experimental evolution approach, monitoring the adaptation to symbiont-conferred protection in sexual populations of the parasitoid L. fabarum over eleven generations. As hosts we used four sublines of a single clone of $A$. fabae that were artificially infected with one of three different isolates of $H$. defensa or symbiont-free as a control. We also tested whether adaptation to symbiont-mediated defenses comes at costs by comparing life-history traits among the evolved populations.

\section{Material and Methods}

\section{INSECTS}

The black bean aphid, Aphis fabae, is a widely distributed agricultural pest reproducing by cyclical parthenogenesis. In Europe, A. fabae uses mainly the spindle tree Euonymus 
europaeus as a primary host for the sexual generation that lays the overwintering eggs. Many species of herbaceous annual plants are used as secondary host for the viviparous, parthenogenetic generations throughout the growth season. It is particularly damaging on broad beans, Vicia faba, and sugar beets, Beta vulgaris (Blackman and Eastop 2006). By keeping it under summer-like conditions with a long photoperiod, it is possible to maintain A. fabae clonally for any period of time in the laboratory.

To test for the adaptation of parasitoids to $H$. defensa-mediated defenses, we used genetically identical aphids with and without different isolates of the symbiont. The common genetic background was a single clone of $A$. fabae, A06-407, that was collected in July 2006 on Chenopodium album in St. Margrethen (Switzerland) and found to be uninfected with any known secondary symbiont of aphids (Vorburger et al. 2009). Since then it was maintained in the laboratory on Vicia faba. Between June 2008 and March 2009 we artificially created three $H$. defens $a$-infected sublines of this clonal host, namely $\mathrm{A} 06-407^{\mathrm{H} 323}, \mathrm{~A} 06-407^{\mathrm{H} 402}$ and $\mathrm{A} 06-407^{\mathrm{H} 76}$, by microinjecting hemolymph from three different A. fabae clones (nrs. A06-76, A06-323 and A06-402) harboring the symbiont and exhibiting different level of resistance to the parasitoid L. fabarum (Vorburger et al. 2009; Rouchet and Vorburger 2012). Using the same genetic background enabled us to separate the protection conferred by $H$. defensa from genetic variation in the aphids' own defenses. Microinjections followed the protocol described in Vorburger et al. (2010) and resulted in stable, heritable infections that were re-confirmed by diagnostic PCR prior to the lines' use in the experiment. Based on partial sequences of two housekeeping genes, $\operatorname{accD}$ (acetyl-CoA carboxylase, $489 \mathrm{bp}$ ) and murE (Murein, $722 \mathrm{bp}$ ), the three isolates of H. defensa belong to two different haplotypes (J.-C. Simon, L. Cayetano and C. 
Vorburger, unpubl. data). $\mathrm{H} 323$ and $\mathrm{H} 402$ have identical sequences that differ from $\mathrm{H} 76$ at both genes, with an overall sequence divergence of $0.99 \%$. We had reason to believe, however, that $\mathrm{H} 323$ and $\mathrm{H} 402$ would differ in their phenotypic effects, because the donor clones from which these two isolates of $H$. defensa were obtained differed strongly in their susceptibility to an asexual line of the parasitoid L. fabarum (see Fig. 1 in Vorburger et al. 2009).

Lysiphlebus fabarum is one of the most abundant aphid parasitoids in Europe and attacks predominantly aphids of the genus Aphis (Starý 2006). There is evidence for further subdivision into host-specific subpopulations within this species (Sandrock and Vorburger 2011; Sandrock et al. 2011). Lysiphlebus fabarum is the main parasitoid of $A$. fabae. In a field experiment, 8051 out of 8725 primary parasitoids collected from $A$. fabae were L. fabarum (L. Rothacher and C. Vorburger, unpubl. data). Together they constitute an ideal system for the study of host-parasitoid interactions mediated by $H$. defensa (Vorburger et al. 2009; Vorburger and Gouskov 2011; Rouchet and Vorburger 2012; Schmid et al. 2012). Lysiphlebus fabarum has the particularity of comprising both sexual (arrhenotokous) and asexual (thelytokous) lineages (Sandrock et al. 2011). Here we worked with sexual L. fabarum. Between May and July 2009, sexuals from eight different locations in Switzerland and in France were collected (Table S1). They were maintained as mass cultures for 6 months on caged colonies of a $H$. defensa-free clone of A. fabae on Vicia faba. Prior to the experiment, 30 females and 20 males from each of the 8 sexual populations were mixed in a large polyester mesh cage $(47.5 \mathrm{~cm} \times 47.5 \mathrm{~cm} \times$ 47.5cm, BugDorm 44545F, MegaView Science, Taichung, Taiwan) and allowed to 
interbreed for three generations. This created the common, genetically variable stock population that we used for the experiment.

\section{EXPERIMENTAL EVOLUTION PROCEDURE}

The experimental evolution procedure consisted of four aphid treatments (i.e. evolution treatments): the $H$. defensa-free clone A06-407 and the three $H$. defensa-infected sublines

of the same clone: A06- $407^{\mathrm{H} 323}$, A06-407 $7^{\mathrm{H} 402}$ and $\mathrm{A} 06-407^{\mathrm{H} 76}$. Each evolution treatment was replicated four times (evolution lines) in independent polyester mesh cages $(24.5 \mathrm{~cm}$ x $24.5 \mathrm{~cm}$ x $24.5 \mathrm{~cm}$, BugDorm-4020F, MegaView Science, Taichung, Taiwan) placed on random positions on an illuminated bench in a climatized room $\left(20^{\circ} \mathrm{C}\right)$. Every cage contained four pots with three broad bean plants colonized by large numbers of aphids from the respective sublines. At the beginning of the experiment, 34 females and 20 males from the stock population were introduced in each cage. Time from oviposition to the emergence of the next generation of wasps is approximately two weeks. When the next generation had emerged, 30 females and 15 males (sometimes fewer, if only low numbers emerged) were transferred into a fresh cage with new aphid colonies from the corresponding treatment sublines. This process was repeated for 11 generations. Through re-stocking the cages with fresh aphids every parasitoid generation, the hosts remained a 'static target', allowing us to observe parasitoid adaptation independent of host or symbiont counter-adaptations.

We first estimated the infectivity of the parasitoids in the mixed parasitoid population at the beginning of the experiment. The infectivity test consisted in exposing a 
parasitoid female to a colony of 48-72h old aphid nymphs from one of the aphid sublines for a period of $12 \mathrm{~h}$, and 11 days later counting the number of mummified hosts, as described by Henter and Via (1995). The proportion of mummified aphids was used as a direct measure of parasitoid infectivity on each of the aphid sublines. Each aphid subline x parasitoid combination was replicated 12 times. At generation 5 and 11, parasitoid infectivity tests were realized the same way with 3 replicates for each aphid subline $\mathrm{x}$ evolution line combination. Moreover, once the experiment was terminated, we mixed the remaining evolution lines within evolution treatments and maintained them for two generations on the $H$. defensa-free clone 407 . The mixing of the evolution lines from the same treatment aimed to reduce the potential effects of bottlenecks and inbreeding in the different evolution lines during the experimental evolution generations. Parasitoid infectivity was then measured based on 8 replicates for each aphid subline $\mathrm{x}$ evolution treatment combination.

\section{CORRELATED RESPONSES}

At the end of the experimental evolution procedure, all evolution lines were placed on $H$. defensa-free clones for two generations before estimating several phenotypic traits to test for correlated responses. Unfortunately, one of the lines that had evolved on A06-407 ${ }^{\mathrm{H} 323}$ was lost at this stage because of a fungal outbreak, leaving three lines of from this treatment to be tested. The individuals to be assayed were produced by placing one parasitoid female in an aphid colony of the H.defensa-free clone A06-407 for 12 hours. This was replicated eight times for each evolution line. Eleven days after the test, the 
number of mummies per colony was counted. Starting from that date, colonies were checked daily and all hatched wasps were collected, providing us with an estimate of development time. All emerging individuals were sexed, weighed to the nearest microgram on a Mettler MX5 microbalance (Mettler Toledo GmbH, Greifensee, Switzerland) and then stored at $-20^{\circ} \mathrm{C}$ for later measurement of the length of both hind tibiae with an ocular micrometer at under a Nikon Eclipse 80i microscope (Nikon, Tokyo, Japon). Thirty-seven females in total were dissected fresh to remove the ovaries and measure the length of 10 mature eggs per female under the microscope to have an estimate of egg size. Apart from egg size, all these traits were measured in the exact same way on parasitoids hatching from the $H$. defensa-free aphid sublines in the infectivity test on the mixed evolution lines (see above). As for infectivity, the mixing of the evolution lines was expected to reduce the potential effects of bottlenecks and inbreeding on these different traits.

\section{STATISTICAL ANALYSIS}

All statistical analyses were carried out in R version 2.11.1 (R Development Core Team 2010). For the infectivity tests, the proportion of aphids exposed to parasitoids that were mummified was taken as the response variable. Overdispersion prevented us from analyzing these success-failure data with a generalized linear models and binomial errors. Hence, the proportions of aphids mummified were arcsine square-root transformed and analyzed with a linear model (LM) or linear mixed models (LMM). First we compared the proportion of aphids mummified by the original stock of parasitoids at generation 0 among aphid sublines with a LM. For the tests after 5 and 11 generations of experimental 
evolution we used LMMs, employing the lmer function from the lme4 package (Bates et al. 2011). We tested for the fixed effects of aphid subline, evolution treatment, and the subline $\mathrm{x}$ treatment interaction, as well as for the random effects of evolution line (nested within evolution treatment) and the evolution line $\mathrm{x}$ aphid subline interaction. $P$-values for the fixed effects were calculated using $F$-tests with Satterthwaite's approximation and p-values for the random effects were calculated based on likelihood-ratio Chi square test with the lmerTest library in R (Kuznetsova et al. 2013). After mixing the evolution lines within treatment at the end of the experiment, we tested for the effects of aphid subline, evolution treatment and the interaction of aphid subline and evolution treatment with a LM.

To test for the effect of evolution treatment on parasitoid infectivity on $H$. defensa-free hosts, we used the results of the infectivity test on the clone A06-407 after mixing evolution lines within evolution treatment at the end of the experiment. We tested for the effect of evolution treatment on parasitoid infectivity (proportion of mummified aphids) with a LM.

To test for potential correlated responses to evolution on symbiont-protected hosts we compared life-history traits (weight, tibia length, development time and egg size) at the end of the experiment with LMMs, again using lmer and lmerTest. We used a mixed model because replicate within evolution line and evolution line (nested within evolution treatment) were considered as random effects. We analyzed the effects on each measured trait of replicate, evolution line, evolution treatment, sex, and the interaction of evolution treatment and sex. A similar analysis was applied to the measurements of life-history traits after mixing of the evolution lines within evolution treatment. 


\section{Results}

\section{EVOLUTION OF INFECTIVITY}

The infectivity assays at generation 0 showed that the stock of parasitoids used as the starting population for the experiment was poorly adapted to $H$. defensa-protected hosts. The mean proportion of hosts mummified differed significantly among aphid sublines $\left(\mathrm{LM}, F_{3,44}=39.80, P<0.001\right)$ and was 0.54 on $H$. defensa-free aphids, but only 0.05 on A06-407 $7^{\mathrm{H} 323}, 0.06$ on $\mathrm{A} 06-407^{\mathrm{H} 402}$ and 0.00 on aphid line A06-407 ${ }^{\mathrm{H} 76}$ (Fig. 1). Not surprisingly, therefore, selection by $H$. defensa-protected aphids was very strong in the first generations of the experiment, and three evolution lines went extinct within 5 generations (two from the $\mathrm{A} 06-407^{\mathrm{H} 76}$ treatment, one from the $\mathrm{A} 06-407^{\mathrm{H} 402}$ treatment). At the next assay in generation 5, an evolutionary response to this selection was already evident in the remaining evolution lines. Infectivity differed significantly among aphid sublines as well as among evolution treatments, and there was a significant aphid subline $\times$ evolution treatment interaction (Table 1), indicating that the infectivities on aphids with or without different isolates of $H$. defensa depended on which aphids the parasitoids had evolved on. Parasitoids from all evolution treatments were able to parasitize the unprotected aphids at similar rates of approximately $40 \%$ (Fig. 1). The parasitoids that had evolved on unprotected aphids (control evolution lines) achieved very low rates of parasitism on all aphid sublines harboring $H$. defensa, similar to the starting population. Parasitoids that had evolved on aphid sublines A06-407 $7^{\mathrm{H} 323}$ and A06-407 ${ }^{\mathrm{H} 402}$ showed a strongly improved ability to parasitize $\mathrm{A} 06-407^{\mathrm{H} 323}$ as well as A06-407 ${ }^{\mathrm{H} 402}$ compared to 
the control lines, but were still near-unable to parasitize A06- $407^{\mathrm{H} 76}$ (Fig. 1). The most infective parasitoids on A06-407 ${ }^{\mathrm{H} 76}$ were from the two remaining evolution lines that had evolved on these strongly protected aphids, but the proportion of aphids mummified of approximately 0.1 was still relatively low, and they also showed low infectivity on the aphids harboring the other two isolates of $H$. defensa (Fig. 1). The evolutionary responses observed at generation 5 were consistent among evolution lines of parasitoids in the same evolution treatment, which was reflected in the non-significant variation among these evolution lines and the lack of an interaction between evolution lines from the same treatment and the aphids they were tested on (Table 1).

Unfortunately, two more evolution lines (both from the A06-407 $7^{\mathrm{H} 402}$ treatment) went extinct between generations 5 and 11 , probably due to the outbreak of a fungal pathogen in their cages. This left us with just a single line of this treatment at the next assay in generation 11, which exhibited rather low infectivity on all aphid sublines except the one it evolved on (Fig. 1). The results for the other evolution treatments were similar to those obtained in generation 5. Wasps evolved on aphids without $H$. defensa still exhibited low infectivites on all aphid sublines possessing $H$. defensa. Wasps evolved on A06- $407^{\mathrm{H} 323}$ had elevated infectivities on $\mathrm{A} 06-407^{\mathrm{H} 323}$ as well as $\mathrm{A} 06-407^{\mathrm{H} 402}$, but still very low infectivity on A06- $407^{\mathrm{H} 76}$. Only the wasps evolved on A06- $407^{\mathrm{H} 76}$ performed well on these aphids (meanwhile approx. $20 \%$ parasitism), but not on the other two aphid sublines harboring $H$. defensa (Fig. 1). Apart from a significant effect of aphid subline, the analyses revealed again a significant aphid subline $\mathrm{x}$ parasitoid treatment interaction (Table 1), confirming that line $\mathrm{x}$ line specificity evolved in the experiment as a consequence of parasitoid adaptation to different isolates of the host's symbionts. As in 
generation 5, there was no significant variation among evolution lines of the same evolution treatment and no evolution line $\mathrm{x}$ aphid subline interaction, showing that the evolutionary response was similar in the different evolution lines of the same treatment. Repeating the infectivity assays after the mixing of the evolution lines from the same evolution treatment, to account for possible inbreeding effects, provided largely comparable results. If anything, there was even stronger evidence for parasitoid adaptation to specific isolates of the symbiont, because all parasitoids that had evolved on aphids harboring $H$. defensa performed best on the very aphid lines they had evolved on compared to the other two protected lines (Fig. 2). This was again reflected in a significant interaction of aphid subline with evolution treatment (Table 1), confirming the strong genotype-by-genotype interaction observed at generation 5 and 11. Although all parasitoids that had adapted to aphids possessing $H$. defensa achieved somewhat lower rates of parasitism on unprotected aphids than those parasitoids that had evolved such aphids (Fig. 2), we did not find a significant effect of the evolution treatment when just analyzing infectivity on $H . d e f e n s a$-free aphids $\left(\mathrm{LM}, F_{3,27}=0.78, P=0.514\right)$. This indicates that parasitoid adaptation to H.defensa-mediated defenses did not decrease parasitoid infectivity on unprotected hosts significantly.

To test specifically if the two $H$. defensa isolates that were indistinguishable based on the sequences of two housekeeping genes also showed identical phenotypic effects, we re-ran our analyses just with the data from the assays on aphid sublines $\mathrm{A} 06-407^{\mathrm{H} 323}$ and A06- $407^{\mathrm{H} 402}$. There was indeed no significant difference in parasitism success by the unselected population at generation $0\left(F_{1,22}=0.025, P=0.876\right)$, and there was neither a significant main effect of aphid line nor a significant aphid subline $\times$ evolution treatment 
interaction at generation $5\left(F_{1,61}=1.601, P=0.211\right.$ and $F_{3,61}=1.737, P=0.169$, respectively) or generation $11\left(F_{1,47}=0.401, P=0.530\right.$ and $F_{3,47}=0.991, P=0.405$, respectively). However, as inspection of Fig. 2 suggests, there was a difference between A06-407 $7^{\mathrm{H} 323}$ and A06-407 $7^{\mathrm{H} 402}$ when wasps were tested after mixing evolution lines within evolution treatment $\left(F_{1,42}=9.856, P=0.003\right)$, as well as a strong aphid subline $\times$ evolution treatment interaction $\left(F_{3,42}=7.570, P<0.001\right)$, reflecting that aphid sublines A06-407 $7^{\mathrm{H} 323}$ and $\mathrm{A} 06-407^{\mathrm{H} 402}$ were most susceptible to the wasps that had evolved on them (Fig. 2).

\section{CORRELATED RESPONSES}

Life-history trait measurements of parasitoids from the evolved lines are provided in Table 2. There was no significant effect of evolution treatment, nor any significant variation among evolution lines within treatment on parasitoid weight or tibia length at the end of the experiment (Table 3). Differences between male and female wasps were not significant, and there were no sex x evolution treatment interactions (Table 3). Egg size did not differ significantly among wasps from the different evolution treatments either (Table 3). The only trait that exhibited significant variation among evolution treatments was development time (Table 3). However, this result has to be interpreted with caution, because it is almost entirely due to a longer development time of wasps evolved in evolution treatment A06-407 ${ }^{\mathrm{H} 402}$ (Table 2). This is the one evolution treatment that was unreplicated at the end of the experiment because three of the four lines in this treatment died out during the experiment (see above). There was also a significant sex 
difference in development time (males emerged slightly later) and a significant evolution treatment x sex interaction because particularly the males of the line evolved on A06$407^{\mathrm{H} 402}$ emerged from mummies very late (Table 3 ). When the analysis was repeated on females only, the variation among evolution treatments was lower, but still nearly $\operatorname{significant}\left(F_{3,43.4}=4.36, P=0.009\right)$.

The second experiment to compare wasp weight, tibia length and development time took place after mixing the remaining evolution lines to account for potential effects of inbreeding. It revealed no significant effect of evolution treatment on any of the three traits (Table 4), supporting that parasitoid adaptation to symbiont-conferred resistance did not entail any marked correlated responses in terms of altered development time or body size.

\section{Discussion}

Experimental evolution is a powerful tool for the study of adaptation. In the present experiment, we found that sexual populations of the parasitoid wasp L. fabarum can adapt quickly to the presence of the defensive endosymbiont $H$. defensa in their aphid host, $A$. fabae. They increased their infectivity on protected aphids within very few generations. These results are concordant with the results of a study by Dion et al. (2011), in which the parasitoid Aphidius ervi experienced a fast increase of infectivity over time when maintained on pea aphids harboring $H$. defensa. By evolving the wasps on aphids harboring different isolates of $H$. defensa in the same genetic background, we could further show that this adaptation was in part specific to the symbiont isolate. The two 
isolates sharing the same sequence types at two housekeeping genes, H323 and H402, represented a similar (but apparently not identical) challenge to the parasitoids. Adaptation to aphid subline A06-407 $7^{\mathrm{H} 323}$ increased infectivity also on $\mathrm{A} 06-407^{\mathrm{H} 402}$ and vice versa, but did not improve the ability to parasitize the host subline $\mathrm{A} 06-407^{\mathrm{H} 76}$. This subline was extremely resistant to the starting population of parasitoids and more difficult for the parasitoids to adapt to. Nevertheless, after 11 generations of experimental evolution on host subline A06-407 ${ }^{\mathrm{H} 76}$, the parasitoids showed a clear increase of infectivity on their own subline, but this did not entail an improved ability to parasitize sublines A06- $407^{\mathrm{H} 323}$ and A06-407 $7^{\mathrm{H} 402}$. Thus, different adaptations appear to be required to overcome host protection by different symbiont strains. What these adaptations are is currently unkown. There is a suggestive observation from the aphid parasitoid A. ervi that behavioral adaptations may play a role. Oliver et al. (2012) found that when confronted with $H$. defensa-protected pea aphids, female wasps were more likely to superparasitize, i.e. inject more than one egg into the host and thus increased their success in parasitizing such aphids. On the other hand, Łukasik et al. (2013) found that parasitoid females tended to avoid oviposition in aphids infected with $H$. defensa. We cannot exclude any behavioral adaptations in our experiment since we did not assay wasp behavior in our experiment, but we currently consider physiological adaptations that increase the tolerance of the parasitoid's egg or larva to the symbiont-conferred defence (presumably toxins, Oliver et al. 2009) more likely.

Regardless of the mechanism, the overall outcome was a significant line $\mathrm{x}$ line interaction on the outcome of infection - the hallmark of a genotype-specific host-parasitoid interaction (Lambrechts et al. 2006). This confirms earlier observations from experiments 
with asexual lines of $L$. fabarum and $H$. defensa-protected aphids which suggested that symbionts mediate the genotype-specificity in this host-parasitoid interaction (Vorburger et al. 2009; Rouchet and Vorburger 2012; Schmid et al. 2012; Cayetano and Vorburger 2013). The presence of $H$. defensa adds an additional, more specific layer of defense in addition to the aphids' own, less specific defenses (Sandrock et al. 2010). The mechanistic basis underlying the observed specificity is still unknown. Differences between $H$. defensa strains of the pea aphid in the level of protection provided to the host have been proposed to be due to differences between APSE bacteriophage variants harbored by the symbiont and known to encode for a variety of toxins (Degnan and Moran 2008). Hence, the strong specificity between symbiont strain and parasitoid line revealed by our study could be explained by differences between symbiont strains in not only the amount of toxins produced, but also in the nature of the toxins.

We found no strong evidence that the adaptation of parasitoids to $H$. defensamediated defenses comes at costs. Wasps evolved on hosts harboring H.defensa experienced no significant decrease in their ability to parasitize symbiont-free hosts. Moreover, we found no consistent differences between evolution treatments in individual mass, size, development time and egg size at the end of the experiment. This contrasts with the experimental evolution experiment by Dion et al. (2011), who reported a decrease in the body size of wasps evolving on $H$. defensa-bearing aphids. However, they compared body sizes of wasps emerging from the hosts they had evolved on and not from a common stock of $H$. defensa-free aphids as in the present experiment. Thus the decreased body size reported in Dion et al. (2011) may have been a direct environmental consequence of developing in aphids with $H$. defensa rather than a correlated genetic 
response of adaptation to such hosts. It is known that wasps developing in aphids harboring $H$. defensa can indeed suffer from negative effects such as delayed development, reduced emergence from mummies and smaller body size (Nyabuga et al. 2010; Schmid et al. 2012). In a selection experiment for increased infectivity of Asobara tabida, a parasitoid of Drosophila melanogaster, Kraaijeveld et al. (2001) also found no differences in the parasitoids' adult size, fat content, egg load and infectivity on susceptible hosts, but they did detect an increase in the duration of the egg stage in the selected lines. This remains one of the sole convincing examples for a cost of increased infectivity in a parasitoid.

Although there are now two studies showing that parasitoids are able to adapt rapidly to $H$. defensa-mediated defenses in aphids (Dion et al. 2011, this work), little is known about how protective symbionts in aphids affect parasitoid populations in the field, e.g. in terms of local adaptation. There is some limited evidence for L. fabarum that at sites with a high prevalence of $H$. defensa in the aphid hosts, parasitoids tend to be more infective on protected hosts, but this relationship was not significant (R. Rouchet et al. unpublished data). The results reported here suggest that parasitoid local adaptation might be more difficult to evolve than envisaged, because rather than just to the presence of $H$. defensa in their hosts, parasitoids may have to adapt to the very strains of symbionts their hosts are harboring. A recent study suggests that strain variation is substantial for $H$. defensa in the pea aphid, more than for any other secondary aphid symbiont, and the P3 gene encoded by APSE in H. defensa shows even higher levels of diversity (Russel et al. 2013). Moreover, factors not considered in our study may also contribute to structuring this variability. In the pea aphid, Acyrthosiphon pisum, host races exist that exploit 
different plants and tend to harbor different genotypes of $H$. defensa (Ferrari et al. 2012; Henry et al. 2013). This raises interesting prospects for future research on ecoevolutionary interactions in such systems, e.g. on the relative roles of host ecology and of the coevolution with parasitoids for symbiont diversity in aphids. The high genetic specificity of the resistance conferred by $H$. defensa observed here and elsewhere certainly suggests that coevolutionary interactions with parasitoids may be highly dynamic, because genotype-specificity readily leads to negative frequency-dependent selection and thus to a rapid turnover of genotypes under antagonistic coevolution.

\section{ACKNOWLEDGMENTS}

We are very thankful to Alexandre Gouskov and Jenny Herzog for help in the laboratory. We also thank Jean-Christophe Simon for sequence typing the $H$. defensa isolates used in this study, as well as two anonymous reviewers and Aneil Agrawal for helpful recommendations to improve our manuscript. This work was supported by the Swiss National Science Foundation.

\section{DATA ARCHIVING}

doi:10.5061/dryad.v8046 


\section{LITERATURE CITED}

Bates, D., M. Maechler, and B. Bolker. 2012. Package 'lme4': Linear mixed-effects model using s4 classes. http://lme4.r-forge.r-project.org/

Brown, K. M., and A. Tellier. 2011. Plant-parasite coevolution: Bridging the gap between genetics and ecology. Annu. Rev. Phytopathol. 49:345-367.

Cayetano, L., and C. Vorburger. 2013. Genotype-by-genotype specificity remains robust to average temperature variation in aphid/endosymbiont/parasitoid system. J. Evol. Biol. 26:1603-1610.

Degnan, P. H., and N. A. Moran. 2008. Evolutionary genetics of a defensive facultative symbiont of insects: exchange of toxin-encoding bacteriophage. Mol. Ecol. 17:916-929.

Dion, E., F. Zélé, J. C. Simon, and Y. Outreman. 2011. Rapid evolution of parasitoids when faced with the symbiont-mediated resistance of their hosts. J. Evol. Biol. $24: 741-750$.

Ebert, D. 2008. Host-parasite coevolution: Insights from the Daphnia-parasite model system. Curr. Opin. Microbiol.11:290-301.

Ferrari, J., A. C. Darby, T. J. Daniell, H. C. J. Godfray, and A. E. Douglas. 2004. Linking the bacterial community in pea aphids with host-plant use and natural enemy resistance. Ecol. Entomol. 29:60-65.

Ferrari, J., J. A. West, S. Via, and H. C. J. Godfray. 2012. Population genetic structure and secondary symbionts in host-associated populations of the pea aphid complex. Evolution 66:375-390. 
Godfray, H. C. J. 1993. Parasitoids Behavioral and Evolutionary Ecology. Princeton University Press: Princeton.

Henry, L. M., J. Peccoud, J.-C. Simon, J. D. Hadfield, M. J. C. Maiden, J. Ferrari, and H. C. J. Godfray 2013. Horizontally transmitted symbionts and host colonization of ecological niches. Curr. Biol. 23:1713-1717.

Henter, H. J. and S. Via. 1995. The potential for coevolution in a host-parasitoid system .1. Genetic-variation within an aphid population in susceptibility to a parasitic wasp. Evolution 49:427-438.

Kraaijeveld, A. R., J. J. M Van Alphen, and H. C. J. Godfray. 1998. The coevolution of host resistance and parasitoid virulence. Parasitology 116: S29-S45.

Kraaijeveld, A. R., K. A. Hutcheson, E. C. Limentani, and H. C. J. Godfray. 2001. Costs of counterdefenses to host resistance in a parasitoid of Drosophila. Evolution $55: 1815-1821$.

Kuznetsova, A., P. B. Brockhoff, and R. H. B. Christensen. 2013. Package 'lmerTest': Tests for random and fixed effects for linear mixed effect models (lmer objects of lme4 package).

Laine, A. L. 2009. Role of coevolution in generating biological diversity - spatially divergent selection trajectories. J. Exp. Bot. 60:2957-2970.

Lambrechts, L., S. Fellous, and J. C. Koella. 2006. Coevolutionary interactions between host and parasite genotypes. Trends Parasitol. 22:12-16.

Łukasik, P., M. van Asch, H. Guo, J. Ferrari, and H. C. J. Godfray. 2013. Unrelated facultative endosymbionts protect aphids against a fungal pathogen. Ecol. Lett. $16: 214-218$ 
Moran, N. A., P. H. Degnan, S. R. Santos, H. E. Dunbar, and H. Ochman. 2005. The players in a mutualistic symbiosis: Insects, bacteria, viruses, and virulence genes. Proc. Natl. Acad. Sci. USA 102:6919-16926.

Nyabuga, F. N., Y. Outreman, J.-C. Simon, D. G. Heckel, and W. W. Weisser. 2010. Effects of pea aphid secondary endosymbionts on aphid resistance and development of the aphid parasitoid Aphidius ervi: a correlative study. Entomol. Exp. Appl. 136:243-253.

Oliver, K. M., J. Campos, N. A. Moran, and M. S. Hunter. 2008. Population dynamics of defensive symbionts in aphids. Proc. R. Soc. B 275:293-299.

Oliver, K. M., P. H. Degnan, G. R. Burke, and N. A. Moran. 2010. Facultative symbionts in aphids and the horizontal transfer of ecologically important traits. Annu. Rev. Entomol 55:247-266.

Oliver, K. M., P. H. Degnan, M. S. Hunter, and N. A. Moran. 2009. Bacteriophages encode factors required for protection in a symbiotic mutualism. Science 325:992994.

Oliver, K. M., N. A. Moran, and M. S. Hunter. 2005. Variation in resistance to parasitism in aphids is due to symbionts not host genotype. Proc. Natl. Acad. Sci. USA 102: 12795-12800.

Oliver, K. M., K. Noge, E. M. Huang, J. M. Campos, J. X. Becerra, and M. S. Hunter. 2012. Parasitic wasp responses to symbiont-based defense in aphids. BMC Biol 10:11. 
Oliver, K. M., J. A. Russell, N. A. Moran, and M. S. Hunter. 2003. Facultative bacterial symbionts in aphids confer resistance to parasitic wasps. Proc. Natl. Acad. Sci. USA 100:1803-1807.

Oliver, K. M., A. H. Smith, and A. Russell. 2013. Defensive symbiosis in the real world advancing ecological studies of heritable, protective bacteria in aphids and beyond. Funct. Ecol. DOI: 10.1111/1365-2435.12133

Rouchet, R., and C. Vorburger. 2012. Strong specificity in the interaction between parasitoids and symbiont-protected hosts. J. Evol. Biol. 25:2369-2375.

Russell, J. A., S. Weldon, A. H. Smith, K. L. Kim, Y. Hu, P. Lukasik, S. Doll, I. Anastopoulos, M. Novin, and K. M. Oliver. 2013. Uncovering symbiont-driven genetic diversity across North American pea aphids. Mol. Ecol. 22:2045-2059.

Sandrock, C., A. Gouskov, and C. Vorburger. 2010. Ample genetic variation but no evidence for genotype specificity in an all-parthenogenetic host-parasitoid interaction. J. Evol. Biol. 23:578-585.

Sandrock, C., B.E. Schirrmeister, and C. Vorburger. 2011. Evolution of reproductive mode variation and host associations in sexual-asexual complex of aphid parasitoids. BMC Evol. Biol. 11:348.

Sandrock, C., and C. Vorburger. 2011. Single-locus recessive inheritance of asexual reproduction in a parasitoid wasp. Curr. Biol. 21:433-437.

Scarborough, C.L., J. Ferrari, and H. C. J. Godfray. 2005. Aphid protected from pathogen by endosymbiont. Science 310:1781-1781. 
Schmid, M., R. Sieber, Y.S. Zimmermann, and C. Vorburger. 2012. Development, specificity and sublethal effects of symbiont-conferred resistance to parasitoids in aphids. Funct. Ecol. 26:207-215.

Starý, P. 2006. Aphid Parasitoids of the Czech Republic (Hymenoptera: Braconidae, Aphidiinae). Academia, Praha.

Thompson, J.N. 1994. The Coevolutionary Process. University of Chicago Press: Chicago.

Vorburger, C., L. Gehrer, and P. Rodriguez. 2010. A strain of the bacterial symbiont Regiella insecticola protects aphids against parasitoids. Biol. Lett. 6:109-111.

Vorburger, C., and A. Gouskov. 2011. Only helpful when required: a longevity cost of harbouring defensive symbionts. J. Evol. Biol. 24:1611-1617.

Vorburger, C., C. Sandrock, A. Gouskov, L. E. Castaneda, and J. Ferrari. 2009. Genotypic variation and the role of defensive endosymbionts in an allparthenogenetic host-parasitoid interaction. Evolution 63:1439-1450.

Windsor, D.A. 1998. Most of the species on Earth are parasites. Int. J. Parasitol. 28:1939-1941.

Woolhouse, M. E. J., J. P. Webster, E. Domingo, B. Charlesworth, and B.R. Levin. 2002. Biological and biomedical implications of the coevolution of pathogens and their hosts. Nature Genet. 32:569-77. 
Figure 1. Mean infectivities of aphid parasitoids (Lysiphlebus fabarum) expressed as the proportion of aphids mummified (successfully parasitized) on four aphid sublines at three different time points of the experiment. Aphid sublines are genetically identical (a single clone of Aphis fabae) but either uninfected with Hamiltonella defensa (-) or experimentally infected with three different isolates of this defensive symbiont $(\mathrm{H} 323$, H402, H76). A: The unselected parasitoid population prior to experimental evolution. B: After 5 generations of experimental evolution. C: After 11 generations of experimental evolution. Means and standard errors in panels B and C were calculated over average infectivities of replicate evolution lines within evolution treatments. Error bars are lacking for evolution treatment $\mathrm{H} 402$ in panel $\mathrm{C}$ because three of the four lines of this treatment had gone extinct by generation 11. 
A Generation 0

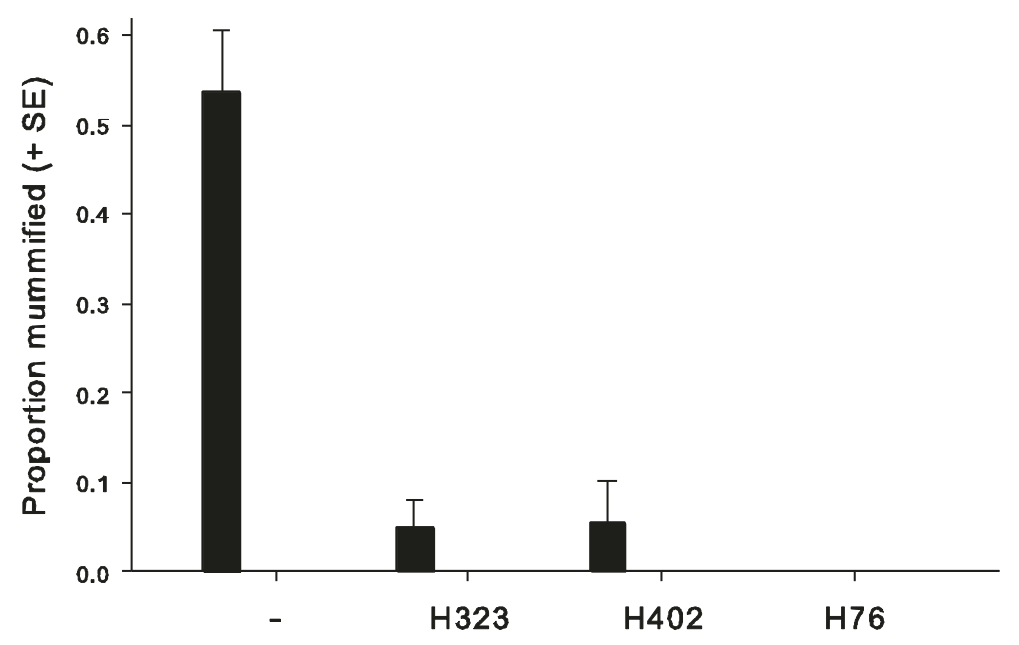

B Generation 5

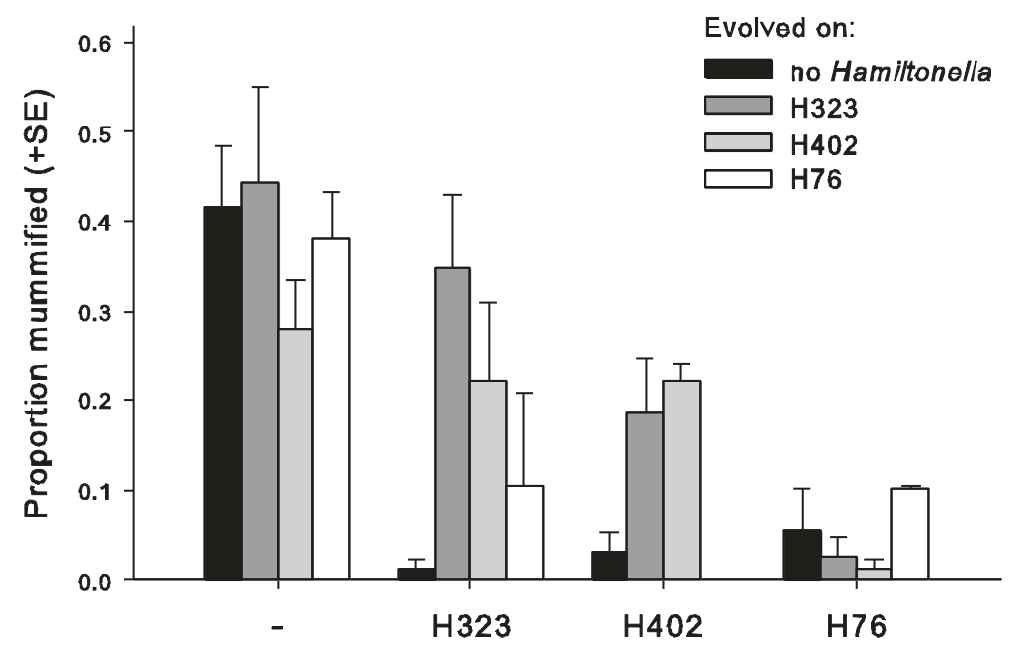

C Generation 11

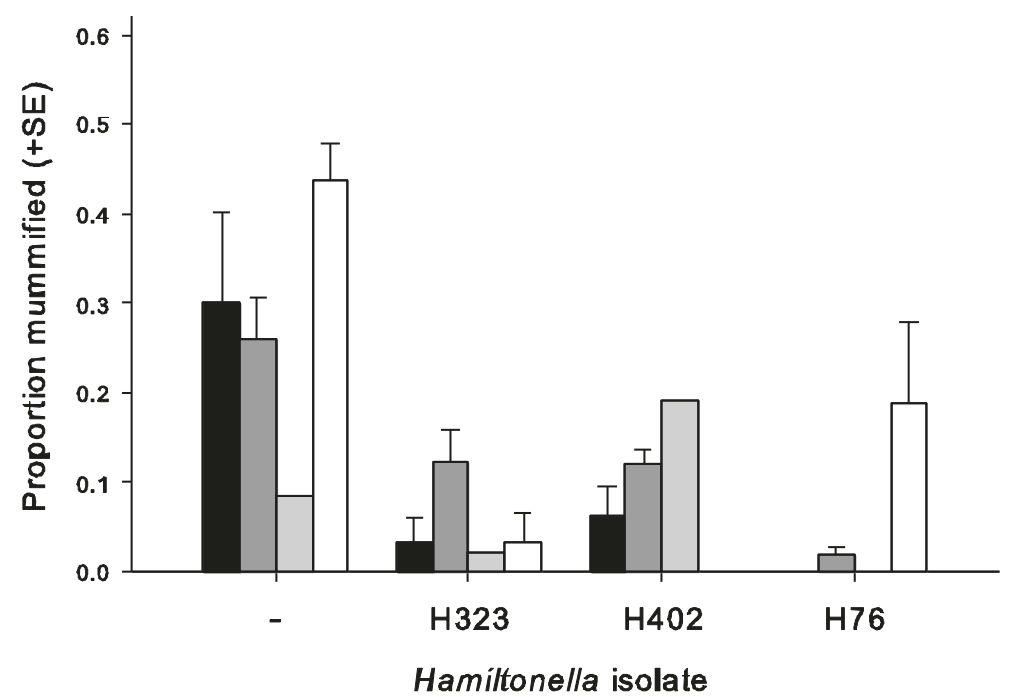


Figure 2. Mean infectivities of aphid parasitoids (Lysiphlebus fabarum) from the four evolution treatments expressed as the proportion of aphids mummified in each of the four aphid sublines, after interbreeding the replicate parasitoid evolution lines within evolution treatment. 


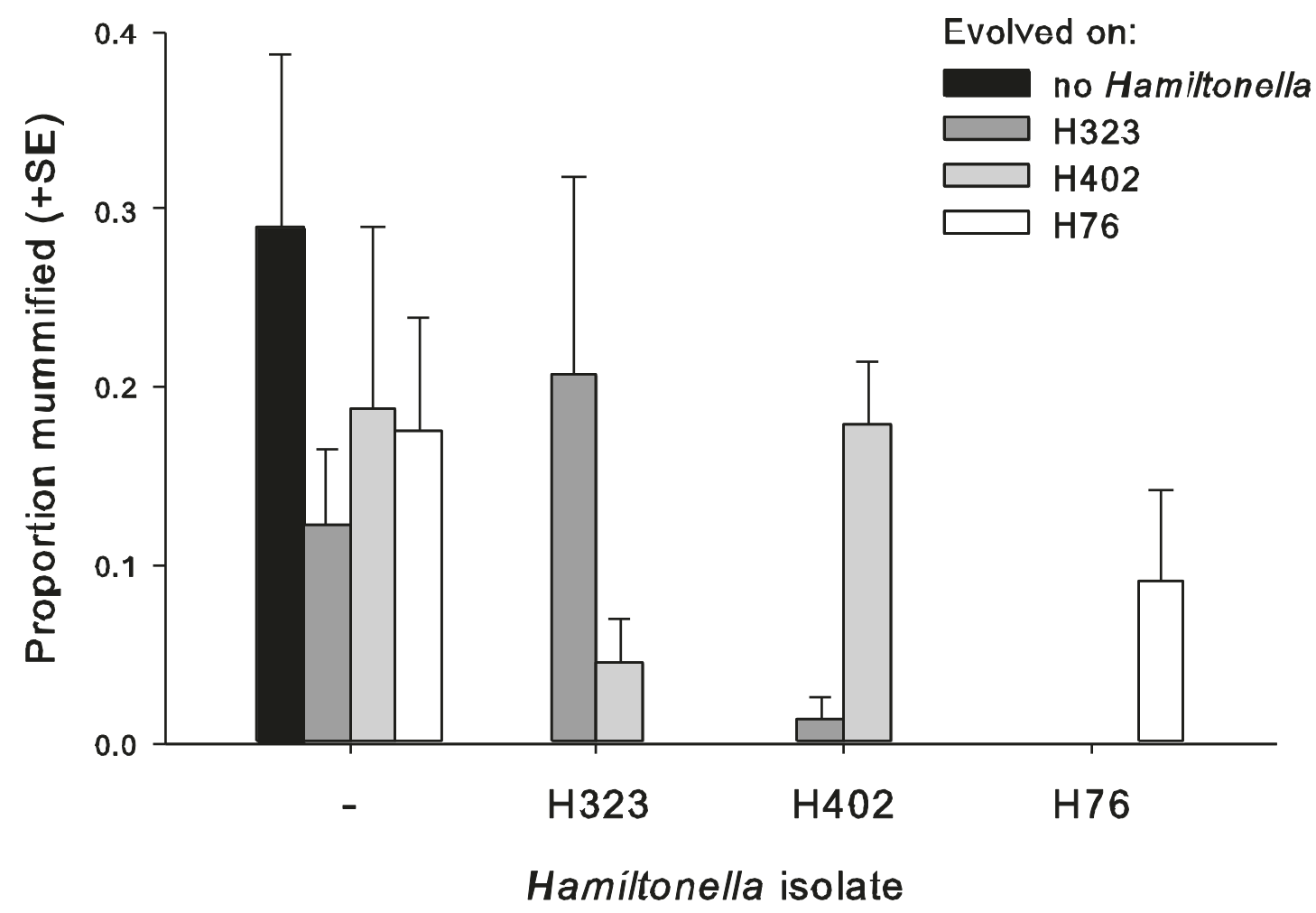


Table 1. Results of linear or linear mixed effects models of the proportion of aphids mummified by parasitoids after 5 and 11 generations of experimental evolution, and after mixing evolution lines within each of the evolution treatments and maintaining them on unprotected hosts for two generations. Proportions were arcsine square-root transformed before analysis. $P$-values of random effects are based on likelihood ratio tests and $P$ values of fixed effects on $F$ tests with Satterthwaite's approximation carried out with the lmerTest library in R (Kuznetsova et al. 2013).

\begin{tabular}{lcclr}
\hline Source & $\begin{array}{l}\text { ndf for } \\
\text { fixed effects }\end{array}$ & $\begin{array}{l}\text { ddf for } \\
\text { fixed effects }\end{array}$ & $\begin{array}{l}F \text { for fixed effects/ } \\
\text { LR } \chi_{1}^{2} \text { for random effects }\end{array}$ & $P$ \\
\hline Generation 5 & & & & $<0.001$ \\
$\quad$ Aphid subline & 3 & 140 & 20.82 & 0.005 \\
Evolution treatment & 3 & 140 & 4.45 & $<0.001$ \\
Aphid subl. x evol. treatment & 9 & 140 & 3.77 & 1.000 \\
Evolution line (treatment) & & & $1.63 \mathrm{e}-09$ & 1.000 \\
Evol. line x aphid subl. & & & $2.42 \mathrm{e}-09$ & $<0.001$ \\
Generation 11 & 3 & 108 & 11.97 & 0.280 \\
Aphid subline & 3 & 108 & 1.30 & 0.030 \\
Evolution treatment & 108 & 2.18 & 1.000 \\
Aphid subl. x evol. treatment & 9 & & 0.00 & 1.000 \\
Evolution line (treatment) & & & $-2.36 \mathrm{e}-10$ & \\
Evol. line x aphid subl. & & & & \\
After mixing evolution lines within evolution treatments & & & \\
Aphid subline & 3 & 93 & 3.494 & 0.019 \\
Evolution treatment & 3 & 93 & 1.778 & $<0.001$ \\
Aphid subl. x evol. treatment & 9 & 93 & 4.135 &
\end{tabular}


Table 2. Parasitoid life-history traits of the different evolution lines assayed after 11 generations of experimental evolution and two generations of relaxed selection on aphids without Hamiltonella defensa. Means and standard errors (in brackets) were calculated over average values from all individuals in a replicate.

\begin{tabular}{lcccc}
\hline Evolution line & $\begin{array}{l}\text { Parasitoid mass } \\
(\mathrm{mg})\end{array}$ & $\begin{array}{l}\text { Tibia length } \\
(\mathrm{mm})\end{array}$ & $\begin{array}{l}\text { Development time } \\
\text { (days) }\end{array}$ & Egg length $(\mu \mathrm{m})$ \\
\hline $\begin{array}{l}\text { (a) after 2 generations of relaxed selection } \\
\text { No Hamiltonella A }\end{array}$ & $0.184(0.010)$ & $0.533(0.011)$ & $11.932(0.120)$ & $116.5(1.3)$ \\
No Hamiltonella B & $0.163(0.008)$ & $0.524(0.010)$ & $12.213(0.088)$ & $122.1(2.8)$ \\
No Hamiltonella C & $0.177(0.004)$ & $0.533(0.005)$ & $12.140(0.058)$ & $122.4(2.8)$ \\
No Hamiltonella D & $0.171(0.009)$ & $0.536(0.011)$ & $11.899(0.231)$ & $115.5(2.6)$ \\
H323 A & $0.166(0.013)$ & $0.502(0.020)$ & $12.012(0.155)$ & $121.4(1.5)$ \\
H323 B & $0.159(0.004)$ & $0.513(0.007)$ & $12.317(0.193)$ & $115.2(2.1)$ \\
H323 D & $0.155(0.010)$ & $0.517(0.009)$ & $12.086(0.182)$ & $118.0(3.2)$ \\
H402 B & $0.185(0.005)$ & $0.539(0.014)$ & $13.384(0.361)$ & $121.8(4.1)$ \\
H76 B & $0.164(0.011)$ & $0.511(0.006)$ & $12.128(0.134)$ & $122.2(0.8)$ \\
H76 C & $0.169(0.003)$ & $0.525(0.003)$ & $12.158(0.064)$ & $113.2(3.9)$ \\
(b) after interbreeding lines within evolution treatment and two generations on Hamiltonella-free hosts \\
No Hamiltonella & $0.207(0.017)$ & $0.553(0.032)$ & $12.560(0.139)$ & \\
H323 & $0.177(0.020)$ & $0.548(0.016)$ & $12.510(0.229)$ & \\
H402 & $0.191(0.007)$ & $0.579(0.008)$ & $12.431(0.181)$ & \\
H76 & $0.170(0.010)$ & $0.551(0.015)$ & $12.821(0.141)$ & \\
\hline
\end{tabular}


Table 3. Results of linear mixed models for parasitoid life-history traits measured in the different evolution lines after 11 generations of experimental evolution and 2 generations of relaxed selection on aphids without Hamiltonella defensa. P-values for the fixed effects were obtained from $F$-tests with Sattherthwaite's approximation and $P$-values for the random effect from likelihood ratio tests.

\begin{tabular}{|c|c|c|c|c|}
\hline Source & $\begin{array}{l}\text { ndf for } \\
\text { fixed effects }\end{array}$ & $\begin{array}{l}\text { ddf for } \\
\text { fixed effects }\end{array}$ & $\begin{array}{c}F \text { for fixed effects/ } \\
\operatorname{LR} \chi_{1}^{2} \text { for random effects }\end{array}$ & $P$ \\
\hline \multicolumn{5}{|l|}{ Weight } \\
\hline Evolution treatment & 3 & 53.0 & 1.998 & 0.125 \\
\hline Sex & 1 & 409.5 & 2.945 & 0.087 \\
\hline Evolution treatment $\mathrm{x}$ sex & 3 & 410.2 & 1.778 & 0.151 \\
\hline Evolution line (treatment) & & & 0.000 & 1.000 \\
\hline Replicate & & & 17.100 & $<0.001$ \\
\hline \multicolumn{5}{|l|}{ Tibia length } \\
\hline Evolution treatment & 3 & 43.6 & 2.285 & 0.092 \\
\hline Sex & 1 & 408.9 & 0.841 & 0.360 \\
\hline Evolution treatment $\mathrm{x}$ sex & 3 & 410.4 & 0.591 & 0.621 \\
\hline Evolution line (treatment) & & & 0.000 & 1.000 \\
\hline Replicate & & & 12.500 & $<0.001$ \\
\hline \multicolumn{5}{|l|}{ Development time } \\
\hline Evolution treatment & 3 & 47.55 & 14.271 & $<0.001$ \\
\hline Sex & 1 & 431.92 & 9.179 & 0.003 \\
\hline Evolution treatment $\mathrm{x}$ sex & 3 & 427.22 & 7.581 & $<0.001$ \\
\hline Evolution line (treatment) & & & 0.000 & 1.000 \\
\hline Replicate & & & 49.100 & $<0.001$ \\
\hline \multicolumn{5}{|l|}{ Egg size } \\
\hline Evolution treatment & 3 & 5.6 & 0.359 & 0.786 \\
\hline Evolution line (treatment) & & & 0.810 & 0.368 \\
\hline
\end{tabular}


Table 4. Results of linear mixed models for parasitoid life-history traits measured after mixing evolution lines within treatment. $P$-values for the fixed effects were obtained from $F$-tests with Sattherthwaite's approximation and $P$-values for the random effect from likelihood ratio tests.

\begin{tabular}{lcccc}
\hline Source & $\begin{array}{c}\text { ndf for fixed } \\
\text { effects }\end{array}$ & $\begin{array}{c}\text { ddf for } \\
\text { fixed effects }\end{array}$ & $\begin{array}{c}F \text { for fixed effects / } \\
\text { LR } \chi_{1}^{2} \text { for random effects/ }\end{array}$ & $P$ \\
\hline Weight & 3 & & & \\
$\quad$ Evolution treatment & 1 & 24.2 & 0.085 & 0.375 \\
$\quad$ Sex & 3 & 75.1 & 0.161 & 0.772 \\
$\quad$ Evolution treatment x sex & & & 7.420 & 0.922 \\
$\quad$ Replicate & & & & 0.006 \\
Tibia lenght & 3 & 24.3 & 0.392 & 0.760 \\
$\quad$ Evolution treatment & 1 & 65.1 & 0.401 & 0.529 \\
Sex & 3 & 66.6 & 0.438 & 0.727 \\
Evolution treatment x sex & & & 0.210 & 0.137 \\
Replicate & & & & \\
Development time & 3 & 23.2 & 0.841 & 0.485 \\
$\quad$ Evolution treatment & 1 & 78.4 & 0.232 & 0.631 \\
Sex & 3 & 75.7 & 0.180 & 0.910 \\
Evolution treatment x sex & & & 9.07 & 0.003 \\
$\quad$ Replicate & & &
\end{tabular}

